\title{
Optimization Models in the Design of Interconnected Regional Power Markets
}

\author{
Alexander M. Malyscheff ${ }^{1 *}$, Milad Javadi ${ }^{2}$, Di Wu ${ }^{3}$, John N. Jiang ${ }^{1}$ and Theodore B. Trafalis ${ }^{4}$ \\ ${ }^{1}$ School of Electrical and Computer Engineering, University of Oklahoma, Norman, Oklahoma 73019, United \\ States \\ ${ }^{2}$ Lower Colorado River Authority, Transmission Services Corporation, Transmission Planning Department, \\ Austin, Texas, United States \\ ${ }^{3}$ Department of Electrical and Computer Engineering, North Dakota State University, Fargo, North Dakota \\ 58108, United States \\ ${ }^{4}$ School of Industrial and Systems Engineering, University of Oklahoma, Norman, Oklahoma 73019, United States \\ Email: malyscheff@gmail.com
}

\begin{abstract}
Determining the impact of dispatched generation in a power system spanning several regions under different jurisdictions presents a variety of challenges to the restructuring of the power industry. In this article several approaches are discussed, which may be useful in an improved design of interconnected regional power markets. In particular, a possible design improvement is presented which provides an insurance against uneven cost distribution by adding additional constraints that bound the costs in each region.
\end{abstract}

Keywords: Seams issues, multi-area optimal power flow, optimization models, net power interchange, power markets.

\section{Introduction}

Power grids and markets interconnect with one another to reduce costs, improve operation, increase overall reliability, and control risks. Specifically, the electric power grids and markets in the United States can be decomposed into independent jurisdictions such as independent system operators (ISOs) and regional transmission organizations (RTOs). A general framework is provided by the Federal Energy Regulatory Commission (FERC) in the form of a standard market design (SMD) which is adopted by all RTOs [1]. Each RTO maximizes its social welfare by solving an optimization problem whose solution provides optimal generation and load levels, power flows, and corresponding nodal prices. Grid structure and bid and offer curves are closely controlled by all jurisdictions. Furthermore, power flows also depend on physical laws; hence, neighboring RTOs may obtain incompatible net power interchanges. The resulting violations of power flow in the coordination of net power interchange are also referred to as seams. Of particular importance in the analysis of seams issues is the net power interchange between independent jurisdictions. Individual jurisdictions have complete information only for their own power market and transmission system. Thus, in addition to operating their own power market, and without having complete information of the adjacent markets, independently interconnected RTOs need to coordinate the power interchanges over the tie-lines. The key challenge to resolving the seams issues is how to manage and price the interchanges. The actual net interchange in real-time can be found by adding all metered interchanges between an RTO and its neighboring control areas. Actual net interchange is given by the sum of scheduled net interchange and inadvertent interchange. Problems of multi-area electric power interchanges determine the amount of dispatched generation in a power system spanning several control areas. A joint optimal dispatch (JOD) represents the most economical dispatch when a group of control areas is considered under a single combined jurisdiction and frequently serves as a reference. Seams issues highlight the inconsistencies that occur when individual RTOs solve simplified multi-area optimal power flow $(\mathrm{OPF})$ problems because information about neighboring control areas is limited. This article discusses some of the challenges surrounding coordinated power interchange and provides ideas how these challenges may be modeled. Assuming a perspective of perfect knowledge, we first show that two interconnected control areas can affect each other's dispatch even in the absence of net power interchange 
over the tie-lines. Next, we consider a situation where both RTOs possess only limited information of each other's power system. We present an optimization model which reconciles the tie-line flows between two control areas. As a result an increase in total dispatch costs of both areas can be observed. This approach may potentially also have applications in other flow problems. Finally, we also provide evidence that the current JOD-based approaches not necessarily guarantee a fair distribution of costs between adjacent control areas. We discuss a variation which provides an insurance against uneven cost distribution by limiting the costs in each control area. After a literature review and background discussion (sections 2 and 3) we will present selected case studies in section 4 illustrating the effect of optimal power flow calculations on neighboring control areas. In section 5 we will then focus on the flow inconsistencies over tie-lines, which represent one aspect of seams issues. In section 6 we will address potential shortcomings of the JOD approach and provide one possible route (by limiting costs) to address these shortcomings. We discuss some thoughts regarding future research in section 7 , while section 8 concludes.

\section{Literature Review}

The existence of so-called seams issues in power systems represents a trading impediment between adjacent control areas.

The recently increasing number of reports on seams issues and dispute filings with FERC underscore the great need to develop a more thorough understanding and effective methodologies to achieve a seamless operation among electric power markets under different jurisdictions [2].

The basic building block in power market operations is the direct current optimal power flow problem $(\mathrm{DCOPF})$, which maximizes social welfare and which establishes as a result generation and load levels for all buses as well as power flows across all transmission lines. Lagrangian multipliers describe the price level for all nodes and are referred to as locational marginal prices (LMPs) or nodal prices. Theoretically, a nation-wide large-scale OPF can be formulated, however, for historical and technical reasons the power system in the United States consists of several regional control areas that are interconnected by so-called tie-lines [3].

Seams issues are tied to inconsistencies which occur because RTOs and ISOs compute an optimal dispatch only for their local jurisdiction. Each RTO and ISO has complete information only for its own control area (e.g. offer curves, network structure); for adjacent regions only limited or no information may be available. A variety of case studies illustrating these inconsistencies were presented in the literature such as those in [4]. For a group of control areas the optimal dispatch across a new "joined" total area is frequently referred to as joint optimal dispatch (JOD). The challenge is to mathematically describe the minimum information exchange between jurisdictions.

Decomposition techniques in mathematical optimization represent a natural way to model optimal power flow over a group of fragmented power systems. Various approaches have been investigated to approximate the JOD solution while keeping in mind that information exchanged between regions should be kept to a minimum. In $[5,6,7]$ Lagrangian relaxation techniques have been investigated to solve multiarea optimal power flow problems. The complicating constraints are included in the Lagrangian, which then becomes separable. An optimal solution is obtained for each control area subproblem followed by an update of the Lagrangian multiplier. The required update of the multiplier is considered a disadvantage of this approach. A method referred to as optimality condition decomposition (OCD) relaxes only the constraints of the neighboring control area, while the local complicating constraint is retained. Here, every subproblem produces an update for both the local decision variables and the local multiplier, which are subsequently distributed to the other subproblems $[8,9,10]$. Extensions of this method have also been presented in $[11,12]$.

A modified coordinated regional dispatch (MCRD) was investigated in [13,14]. The capacity of transmission lines is split between RTOs and iteratively adjusted according to the price that each RTO is willing to pay for that capacity.

A marginal equivalent (ME) approach was presented in $[15,16]$. Note that the aforementioned studies identify the joint optimal dispatch (JOD) solution. Whether one or more control areas will be at an economic disadvantage under JOD was not included in these investigations. 
Among practitioners the notion of proxy buses is well-established [4]. Both PJM and NYISO use proxy buses to model and price scheduled net interchange with their adjacent control areas [17]. ISO New England reserves individual proxy buses for each of its neighboring control areas [18].

Some RTOs adjust the power flow between control areas on an hourly basis. The authors in [19] present a method to estimate the secure range for interchange adjustment.

\section{Background}

Mathematically, a power transmission system can be modeled as a network or graph $\mathcal{G}(\mathcal{N}, \mathcal{L})$ where the set of nodes is given by $\mathcal{N}=\{n: n=1, \cdots, N\}$ and a set of $M$ edges or transmission lines are denoted by $\mathcal{L}=\{(i, j): i, j \in \mathcal{N}, i \neq j\}$. Power generated at node $n$ is denoted by $P_{n}$, or $\mathbf{P}^{T}=\left(P_{1}, \cdots, P_{N}\right)$ in vector format. Upper and lower bounds for scalars and vectors are given by $\bar{P}_{n}$ and $\underline{P}_{n}$ as well as $\overline{\mathbf{P}}$ and $\underline{\mathbf{P}}$. The phase angle at node $n$ is given by $\Theta_{n}$ and $\boldsymbol{\Theta}^{T}=\left(\Theta_{1}, \cdots, \Theta_{N}\right)$. The power flow over an individual transmission line from bus $i$ to bus $j$ is denoted by $T_{i, j}$ with $\mathbf{T}_{M}^{T}=\left\{T_{i, j}:(i, j) \in \mathcal{L}, i \leq j\right\}$. The upper bound on the power flow (capacity) between buses $i$ and $j$ is given by $\bar{T}_{i, j}$ ( $\overline{\mathbf{T}}$ in vector form). Firm injection and withdrawal flows are labeled $\left(\mathbf{F}^{G}\right)^{T}=\left(F_{1}^{G}, \cdots, F_{N}^{G}\right)$ and $\left(\mathbf{F}^{L}\right)^{T}=\left(F_{1}^{L}, \cdots, F_{N}^{L}\right)$, respectively. Firm net flows are given by $\mathbf{F}=\mathbf{F}^{G}-\mathbf{F}^{L}$. Individual transmission line susceptances between buses $i$ and $j$ are given by $B_{i, j}$. Unless otherwise stated we shall assume $B_{i, j}=1000 \Omega^{-1}$. The bus susceptance matrix $\mathbf{Y} \in \Re^{N}$ encodes the structure of the transmission network in compact form. A matrix $\mathbf{K} \in \Re^{M \times N}$ maps the phase angles at nodes $i$ and $j$ to the power flow $T_{i, j}$. Let $\mathbf{c}^{T}=\left(c_{1}, \cdots, c_{N}\right) \in \Re^{N}$ denote the price of offered generation per unit $(\mathrm{MWh})$. With the reference node labeled $\rho$ the direct current optimal power flow (DCOPF) model can be written as problem $\left(\mathcal{P}_{1}\right)$ :

$$
\begin{aligned}
& \min _{\mathbf{P}, \boldsymbol{\Theta}} \mathbf{c}^{T} \mathbf{P} \quad\left(\mathcal{P}_{1}\right) \\
& \text { subject to: } \\
& \mathbf{P}+\mathbf{F}^{G}-\mathbf{F}^{L}-\mathbf{Y} \boldsymbol{\Theta}=\mathbf{0}_{N}, \quad(\boldsymbol{\Lambda}) \\
&-\overline{\mathbf{T}} \leq \mathbf{K} \boldsymbol{\Theta} \leq \overline{\mathbf{T}} \\
&-\underline{\mathbf{P}} \leq \mathbf{P} \leq \overline{\mathbf{P}} \\
&-\pi \mathbf{1} \leq \boldsymbol{\Theta} \leq \pi \mathbf{1} \\
& \Theta_{\rho}=0 .
\end{aligned}
$$

The objective function (1) minimizes all generation cost, which is identical to maximizing social welfare under firm loads. The expression in (2) reflects Kirchhoff's nodal power flow constraints, while (3) impose capacity limits over the appropriate transmission lines. In (4) generator limits are specified. The box constraints in (5) restrict the phase angles at all buses. Finally, (6) fixes the phase angle at the reference node. We also introduce Lagrangian multipliers associated with all constraints. Specifically, $\boldsymbol{\Lambda}^{T}=\left(\lambda_{1}, \cdots, \lambda_{N}\right)$ in (2) describes the set of locational marginal prices (LMPs) at node $i$. The optimal solution to model $\left(\mathcal{P}_{1}\right)$ is given by $\mathbf{P}^{*}$ and $\boldsymbol{\Theta}^{*}$ with the corresponding objective function value of $Z^{*}=\mathbf{c}^{T} \mathbf{P}^{*}$.

One objective of this paper is to illustrate how DC optimal power flow in one control area is affected by changes in operating conditions in an adjacent control area. Using a simple example we demonstrate that power traversing an adjacent control area affects generation costs and congestion in a given control area, even when the net power interchange over the tie-lines connecting the two areas is zero. Furthermore a model to reconcile flow over tie-lines is suggested and potential shortcomings of the JOD solution are described. Specifically, we would like to point out that the JOD-based approach might violate some economic principles. While these seams issues can be discussed using real-world large-scale models, we decided that the details of these discussions are best presented using simplified models that can be more easily analyzed using graphs. In some instances a generalization to more comprehensive power systems may be straightforward. Hence, in the remaining sections of this paper we will focus for the most part on a simplified network model with $N=6$ buses and $M=8$ transmission lines as shown in Figure 1 . The power system in Figure 1 may contain up to six generators $P_{n}, n=1, \cdots, 6$. Similarly, up to six firm injection or withdrawal flows are indicated by $F_{n}, n=1, \cdots, 6$. 


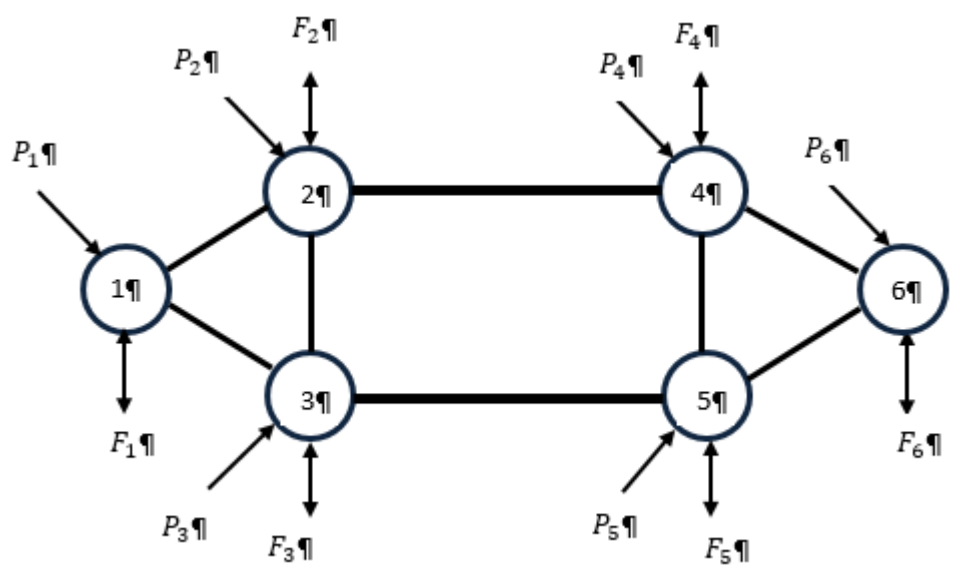

Figure 1. A simple network

\section{Interchanges between Adjacent Control Areas}

\subsection{Examples Illustrating the Interchange Impact}

In this subsection several examples will be presented to illustrate how activities in one control area can influence the dispatching decisions in a neighboring control area. In particular, we show how the dispatching decisions in an adjacent jurisdiction can lead to congested lines and an increase in generation costs elsewhere even though no net power interchange between RTOs occurs. We begin by discussing three cases based on the network shown in Figure 1. Buses 1 to 3 represent RTO A, buses 4 to 6 describe RTO B. RTO A and B are connected over two tie-lines given by $(2,4)$ and $(3,5)$. In the following we assume the perspective of RTO A. Consider the following power flow model shown in Figure 2. Two generators at

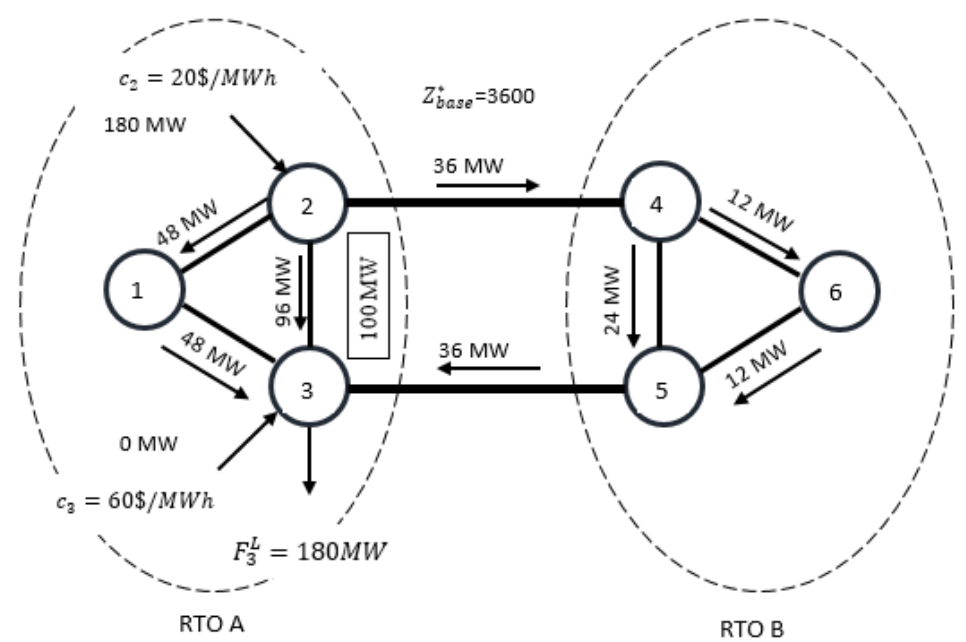

Figure 2. No power flow transiting RTO B

nodes 2 and 3 offer power at a price of $\$ 20 / \mathrm{MWh}$ and $\$ 60 / \mathrm{MWh}$, while a firm load of 180 MW must be served at node 3 . The capacity at line $(2,3)$ is limited to $\bar{T}_{2,3}=100 M W$. No generation occurs in RTO B and no loads must be served here. We label this extreme scenario as the base case. The optimal solution for this case is given by $\left(\mathbf{P}_{\text {base }}^{*}\right)^{T}=(0,180,0,0,0,0),\left(\boldsymbol{\Theta}_{\text {base }}^{*}\right)^{T}=(0,0.048,-0.048,0.012,-0.012,0)$. For 
the objective function value, that is, the minimal cost of generation one finds $Z_{b a s e}^{*}=3600$. The optimal power flows are also depicted in Figure 2. The majority of generation is being routed through RTO A. However a power flow of $36 \mathrm{MW}$ passes through RTO B. A flow of $36 \mathrm{MW}$ can be observed over the tie-line from bus 2 to 4, while a flow toward RTO A is being registered between buses 5 and 3. Note that the net power interchange between the control areas is zero and that the line $(2,3)$ is not at its limit.

Next, consider the case where a power flow of $280 \mathrm{MW}$ is transiting through RTO B. More precisely, we have $F_{4}^{G}=280 M W$ and $F_{5}^{L}=280 M W$ as shown in Figure 3. All other parameters are left unchanged. Optimal generation is given by $\left(\mathbf{P}_{4 \rightarrow 5}^{*}\right)^{T}=(0,117.5,62.5,0,0,0),\left(\Theta_{4 \rightarrow 5}^{*}\right)^{T}=$ $(0,0.050,-0.050,0.083,-0.083,0)$ while the phase angles are described by . Compared to the previous case the objective function value increases to $Z_{4 \rightarrow 5}^{*}=6100$. The detailed solution is provided in

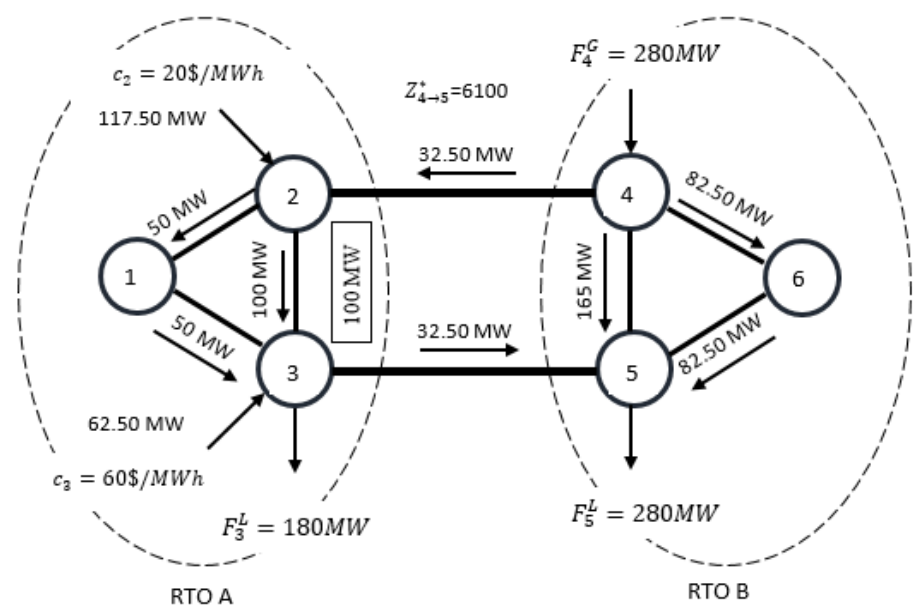

Figure 3. Power flow transiting RTO B from node 4 to node 5

Figure 3. With a power flow of $280 \mathrm{MW}$ transiting RTO B from node 4 to node 5 a flow of $32.50 \mathrm{MW}$ is routed through RTO A. This additional power flow precipitates congestion at line $(2,3)$. Congestion at line $(2,3)$ in turn prohibits RTO A from serving the load at node 3 using cheap generation at node 2. Instead, generation at node 2 is reduced from $180 \mathrm{MW}$ to $117.50 \mathrm{MW}$, while generation at node 3 is increased from $0 \mathrm{MW}$ to $62.5 \mathrm{MW}$. Shifting generation from node 2 to node 3 increases cost by 2500 .

Next, the power flow traversing RTO B is reversed; a flow of $280 \mathrm{MW}$ is injected at node 5 and withdrawn from node 4 as illustrated in Figure 4 . The optimal power dispatch is now calculated as $\left(\mathbf{P}_{5 \rightarrow 4}^{*}\right)^{T}=$ $(0,180,0,0,0,0)$ while the phase angle vector is described by $\left(\boldsymbol{\Theta}_{5 \rightarrow 4}^{*}\right)^{T}=(0,0.029,-0.029,-0.063,0.063,0)$. The objective function value is given by $Z_{5 \rightarrow 4}^{*}=3600$. Figure 4 displays the resulting power flows. With a power flow of $280 \mathrm{MW}$ entering RTO B at node 5 a power flow of $92.00 \mathrm{MW}$ from control area B is diverted to satisfy the load at RTO A's node 3. Simultaneously, generation in RTO A at node 2 is transmitted over the tie-lines toward RTO B satisfying load at node 4. A combination where loads of one control area are served by generators of a neighboring control area may be interpreted as a "symbiotic" network structure. A consequence of this structure is that line $(2,3)$ is not congested and the load at node 3 can be satisfied entirely by the generator at node $2(180 \mathrm{MW})$. While the net flow over the tie-lines remains zero, the flows on the individual lines are reversed compared to the previous case. Since RTO A has full knowledge of all activities in RTO B, including the network structure, the cases discussed in this subsection represent an instance of JOD modeling. Table 1 provides an overview over the discussed cases.

\subsection{Results with Realistic Model}

In this subsection we further illustrate our analysis with a close-to-real-world power system model. The entire interconnection includes 12,078 buses, 814 generators, and over 14,000 transmission lines, branches 


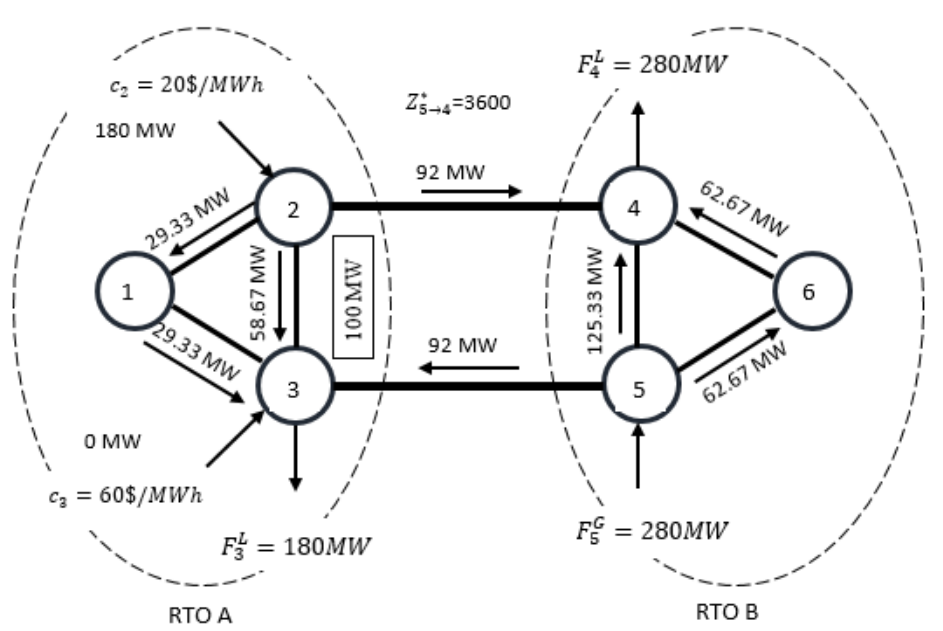

Figure 4. Power flow transiting RTO B from node 5 to node 4

Table 1. Results of subsection 4.1

\begin{tabular}{ccccc}
\hline $\begin{array}{c}\text { Injection bus 4 / } \\
\text { Withdrawal bus } 5\end{array}$ & Line $(2,4)$ & Line $(3,5)$ & $\begin{array}{c}\text { Line }(2,4) \\
\text { congested }\end{array}$ & $Z^{*}$ \\
\hline $0 \mathrm{MW}$ & $+36.00 \mathrm{MW}$ & $+36.00 \mathrm{MW}$ & NO & 3,600 \\
$280 \mathrm{MW}$ & $-32.00 \mathrm{MW}$ & $-32.00 \mathrm{MW}$ & YES & 6,100 \\
$-280 \mathrm{MW}$ & $+92.00 \mathrm{MW}$ & $+92.00 \mathrm{MW}$ & NO & 3,600 \\
\hline
\end{tabular}

and transformers. In this system we define two regions to mimic the two control areas previously defined as RTO A and RTO B. Region A contains approximately 1,000 buses, 67 generators, and 1,316 lines, while the larger region $\mathrm{B}$ comprises the remaining grid elements. The two regions are interconnected through 98 tie-lines. In these experiments we focus on the actual net interchange without any scheduled interchange between the regions, that is, generation and load are balanced in both region A and region B. Following subsection 4.1 the net interchange between two regions is observed under different operating conditions. Specifically, we consider three cases with the same amount of total load in region A balanced at 6,000 MW; in the first case, no load or generation occurs in region B. For the second case, region B is exposed to balanced generation and load. In the last case, generation and load are balanced in both regions as in the second case; however, load and generation in region B have been reduced by $16 \%$. Table 2 displays the results. The results of experiments with a large-scale power system model presented in

Table 2. Results of subsection 4.2

\begin{tabular}{cccc}
\hline Case & $\begin{array}{c}\text { Exports from RTO A } \\
\text { (positive) }\end{array}$ & $\begin{array}{c}\text { Imports from RTO B } \\
\text { (negative) }\end{array}$ & $\begin{array}{c}\text { Net interchange } \\
\text { flow }\end{array}$ \\
\hline Case 1 & $+405.80 \mathrm{MW}$ & $-405.80 \mathrm{MW}$ & $0.000 \mathrm{MW}$ \\
Case 2 & $+1266.63 \mathrm{MW}$ & $-1266.84 \mathrm{MW}$ & $-0.207 \mathrm{MW}$ \\
Case 3 & $+1053.60 \mathrm{MW}$ & $-1053.64 \mathrm{MW}$ & $-0.038 \mathrm{MW}$ \\
\hline
\end{tabular}

Table 2 confirm the observations from subsection 4.1. In all three cases the net interchanges are close to zero. Put differently, for all operating conditions exports from region A to region B are approximately equal and opposite in sign to the imports from region $\mathrm{B}$ to region $\mathrm{A}$, when both region $\mathrm{A}$ and region $\mathrm{B}$ are balanced. It is also confirmed that the magnitude of the exports and imports varies under varying 
operating conditions in region $\mathrm{B}$. This is a consequence of region B being exposed to different generation and load patterns. In this experiment we also calculated the operating costs and observed that they are different for all three cases. This observation implies that the generation and load pattern in region $\mathrm{A}$ is affected by the operating condition in region $\mathrm{B}$. In addition to the economic impact the operating condition in region B may also influence the reliability of region A, as the calculated voltage profiles and reactive power margins are changed for all three cases.

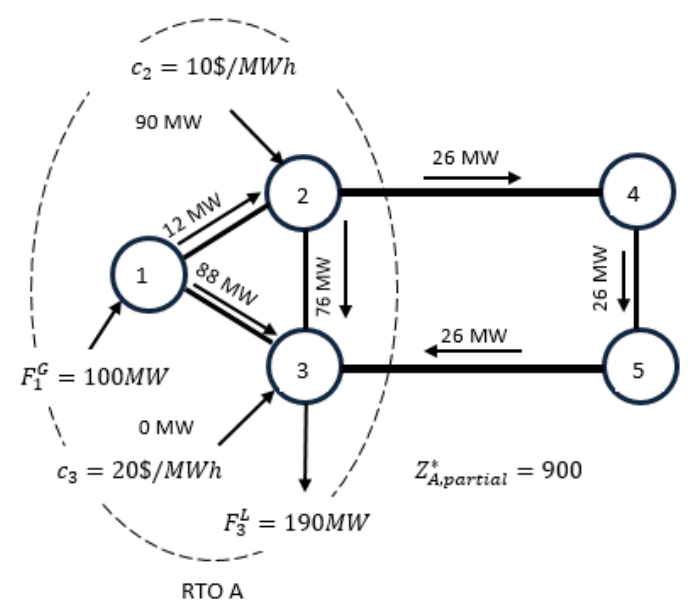

Figure 5. Flow pattern in RTO A with partial separation

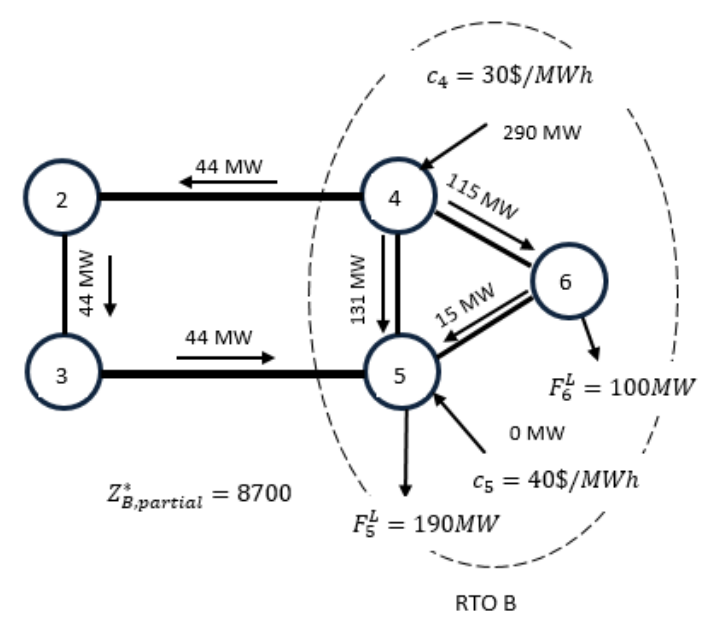

Figure 6. Flow pattern in RTO B with partial separation

\section{A Model to Resolve Inconsistent Tie-Line Flows}

\subsection{Inconsistent Tie-Line Power Flows}

In section 4 the optimal dispatch was computed assuming that all relevant information was available to individual RTOs. In subsections 5.1 and 5.2 we will restrict that information. As a result the power 
flow over the tie-lines will depend on which RTO is carrying out the computation, leading eventually to inconsistent solutions. For the purpose of the discussion in this document we exclude scheduled interchange at this stage. To begin, consider a situation where RTO A no longer has full information access to RTO B's transmission network. Furthermore, in contrast to section 4 the optimal power flow solution of RTO $\mathrm{B}$ will also be taken into consideration. The two RTOs will obtain different solutions for their respective dispatches leading to an inconsistent estimation of power flows over the tie-lines. Specifically, both RTOs only possess knowledge of the buses involving the tie-lines. In addition to its own market and transmission network RTO A has knowledge only of buses 4 and 5 (see Figure 5). Generation in RTO A occurs at nodes 2 and 3 offering power at $\$ 10 / \mathrm{MWh}$ and $\$ 20 / \mathrm{MWh}$ respectively. Firm power of $F_{1}^{G}=100 M W$ is injected in node 1 , a withdrawal of $F_{3}^{L}=190 M W$ occurs at node 3 . RTO B's information is limited to its own market and network together with buses 2 and 3 in RTO A (see Figure 6). RTO B has two generators at nodes 4 and 5 at prices $\$ 30 / \mathrm{MWh}$ and $\$ 40 / \mathrm{MWh}$ respectively. Firm power is withdrawn at nodes 5 and $6\left(F_{5}^{L}=190 M W, F_{6}^{L}=100 M W\right)$. Neither RTO A or B have any capacitated lines, and all generators have an output limit of $\bar{P}_{i}=300 M W, \forall i=2,3,4,5$ (see Figures 5 and 6 ). RTO A solves the optimization problem:

$$
\min _{\mathbf{P}_{A^{\prime}}, \Theta_{A^{\prime}}} \mathbf{c}_{A^{\prime}}^{T} P_{A^{\prime}} \quad\left(\mathcal{P}_{2}\right)
$$

subject to:

$$
\begin{aligned}
\mathbf{A}_{A^{\prime}} \mathbf{x}_{A^{\prime}}+\mathbf{b}_{A^{\prime}} & =\mathbf{0} & \\
0 \leq P_{i} & \leq 300, & \forall i=1, \cdots, 5 \\
-\pi \leq \Theta_{i} & \leq \pi, & \forall i=1, \cdots, 5 \\
\Theta_{1} & =0, &
\end{aligned}
$$

where

$$
\tilde{\mathbf{A}}_{A^{\prime}}=\left[\begin{array}{lllll}
0 & 0 & 0 & 0 & 0 \\
0 & 1 & 0 & 0 & 0 \\
0 & 0 & 1 & 0 & 0 \\
0 & 0 & 0 & 0 & 0 \\
0 & 0 & 0 & 0 & 0
\end{array}\right]
$$

and

$$
\overline{\mathbf{A}}_{A^{\prime}}=\left[\begin{array}{ccccc}
-2000 & 1000 & 1000 & 0 & 0 \\
1000 & -3000 & 1000 & 1000 & 0 \\
1000 & 1000 & -3000 & 0 & 1000 \\
0 & 1000 & 0 & -2000 & 1000 \\
0 & 0 & 1000 & 1000 & -2000
\end{array}\right]
$$

such that

$$
\mathbf{A}_{A^{\prime}}=\left[\begin{array}{ll}
\tilde{\mathbf{A}}_{A^{\prime}} & \overline{\mathbf{A}}_{A^{\prime}}
\end{array}\right]
$$

Furthermore,

$$
\begin{aligned}
& \mathbf{b}_{A^{\prime}}^{T}=\left(\begin{array}{lllll}
F_{1} & 0 & F_{3} & 0 & 0
\end{array}\right) \\
& \mathbf{c}_{A^{\prime}}^{T}=\left(\begin{array}{lllll}
0 & 10 & 20 & 0 & 0
\end{array}\right)
\end{aligned}
$$

and

$$
\begin{aligned}
& \mathbf{x}_{A^{\prime}}^{T}=\left(\mathbf{P}_{A^{\prime}}^{T} \Theta_{A^{\prime}}^{T}\right) \\
& \mathbf{P}_{A^{\prime}}^{T}=\left(\begin{array}{lllll}
P_{1} & P_{2} & P_{3} & P_{4} & P_{5}
\end{array}\right) \\
& \Theta_{A^{\prime}}^{T}=\left(\begin{array}{lllll}
\Theta_{1} & \Theta_{2} & \Theta_{3} & \Theta_{4} & \Theta_{5}
\end{array}\right) \text {. }
\end{aligned}
$$

The resulting solution is given by $\left(\mathbf{P}_{A, \text { partial }}^{*}\right)^{T}=(0,90,0,0,0)$ and $\left(\boldsymbol{\Theta}_{A, \text { partial }}^{*}\right)^{T}=$ $(0,-0.012,-0.088,-0.037,-0.063)$ with an objective function value of $Z_{A, p a r t i a l}^{*}=900$. Along with the market parameters Figure 5 also displays the optimal power flows from the perspective of RTO A. In particular, a clock-wise power flow of $26 \mathrm{MW}$ traversing the path 2-4-5-3 can be observed. Next, consider 
the optimization problem from the perspective of the neighboring RTO B. Observe the different indexing for RTO A $(n=1, \cdots, 5)$ and RTO B $(n=2, \cdots, 6)$.

$$
\begin{aligned}
& \min _{\mathbf{P}_{B^{\prime \prime}}, \boldsymbol{\Theta}_{B^{\prime \prime}}} \mathbf{c}_{B^{\prime \prime}}^{T} P_{B^{\prime \prime}} \quad\left(\mathcal{P}_{3}\right) \\
& \text { subject to: } \\
& \mathbf{A}_{B^{\prime \prime}} \mathbf{x}_{B^{\prime \prime}}+\mathbf{b}_{B^{\prime \prime}}=\mathbf{0} \\
& 0 \leq P_{i} \leq 300, \quad \forall i=2, \cdots, 6 \\
&-\pi \leq \Theta_{i} \leq \pi, \quad \forall i=2, \cdots, 6 \\
& \Theta_{6}=0,
\end{aligned}
$$

where

$$
\tilde{\mathbf{A}}_{B^{\prime \prime}}=\left[\begin{array}{lllll}
0 & 0 & 0 & 0 & 0 \\
0 & 0 & 0 & 0 & 0 \\
0 & 0 & 1 & 0 & 0 \\
0 & 0 & 0 & 1 & 0 \\
0 & 0 & 0 & 0 & 0
\end{array}\right]
$$

and

$$
\overline{\mathbf{A}}_{B^{\prime \prime}}=\left[\begin{array}{ccccc}
-2000 & 1000 & 1000 & 0 & 0 \\
1000 & -2000 & 0 & 1000 & 0 \\
1000 & 0 & -3000 & 1000 & 1000 \\
0 & 1000 & 1000 & -3000 & 1000 \\
0 & 0 & 1000 & 1000 & -2000
\end{array}\right]
$$

such that

$$
\mathbf{A}_{B^{\prime \prime}}=\left[\tilde{\mathbf{A}}_{B^{\prime \prime}} \overline{\mathbf{A}}_{B^{\prime \prime}}\right]
$$

Furthermore,

$$
\begin{aligned}
& \mathbf{b}_{B^{\prime \prime}}^{T}=\left(\begin{array}{llll}
0 & 0 & 0 & -F_{5}-F_{6}
\end{array}\right) \\
& \mathbf{c}_{B^{\prime \prime}}^{T}=\left(\begin{array}{lllll}
0 & 0 & 30 & 40 & 0
\end{array}\right)
\end{aligned}
$$

and

$$
\begin{aligned}
& \mathbf{x}_{B^{\prime \prime}}^{T}=\left(\mathbf{P}_{B^{\prime \prime}}^{T} \Theta_{B^{\prime \prime}}^{T}\right) \\
& \mathbf{P}_{B^{\prime \prime}}^{T}=\left(\begin{array}{lllll}
P_{2} & P_{3} & P_{4} & P_{5} & P_{6}
\end{array}\right) \\
& \boldsymbol{\Theta}_{B^{\prime \prime}}^{T}=\left(\Theta_{2} \Theta_{3} \Theta_{4} \Theta_{5} \Theta_{6}\right) \text {. }
\end{aligned}
$$

The optimal generation in control area B is given by $\left(\mathbf{P}_{B, \text { partial }}^{*}\right)^{T}=(0,0,290,0,0)$; for the optimal phase angles one obtains $\left(\boldsymbol{\Theta}_{B, \text { partial }}^{*}\right)^{T}=(0.0718,0.0282,0.1155,-0.0155,0)$. The cost of generation in RTO B is given by $Z_{B \text {,partial }}^{*}=8700$. The detailed generation levels and power flows are shown in Figure 6 . We also record the total dispatching cost from both areas. We find $Z_{\text {partial }}^{*}=Z_{A, \text { partial }}^{*}+Z_{B, \text { partial }}^{*}=$ $900+8700=9600$. Observe that the optimal solution for RTO B is given by a counter-clockwise power flow of $44 \mathrm{MW}$ along a path comprising the nodes 4-2-3-5. Recall that RTO A's solution includes a clockwise flow along the path 2-4-5-3. Obviously, the two power flows over the tie-lines $(2,4)$ and $(3,5)$ can not occur both simultaneously. This inconsistency in the estimation of flows represents a typical incident of a seams issue and occurs frequently in practice. This discrepancy is generally resolved using ad-hoc methods and operator's experience as well as complicated control or adjustments such as those carried out by automatic generation control (AGC) systems. However, the resulting flows, after ad-hoc or automated control corrective measures, are difficult to predict. These unpredictable flows are often referred to as inadvertent interchange, which occur even without net scheduled interchanges. Note also that ad-hoc resolution may also compromise the economic efficiency of the dispatch or the grid reliability. Next, we suggest a mathematical approach that may help reconcile interchanges over edges of "quasi-disjoint" networks. 


\subsection{A Formulation to Resolve Inconsistent Flows}

Next, we will present an approach to resolve the inconsistency observed in subsection 5.1. In order to do so, observe that the problems described in Figures 5 and 6 can be treated as two completely separate optimization problems. Consequently, they can be combined into a single optimization problem and solved in parallel. The resulting solution would be identical to the ones observed in subsection 5.1. However, we are free to include additional constraints in this combined model. In terms of notation we shall label the phase angles corresponding to RTO A (Figure 5) as prime (') and the phase angles corresponding to RTO B (Figure 6) as double prime $\left({ }^{\prime \prime}\right)$. We now impose similarity of the flows over the tie-lines using the phase angles, i.e., we include $\Theta_{2}^{\prime}-\Theta_{4}^{\prime}=\Theta_{2}^{\prime \prime}-\Theta_{4}^{\prime \prime}$ and $\Theta_{3}^{\prime}-\Theta_{5}^{\prime}=\Theta_{3}^{\prime \prime}-\Theta_{5}^{\prime \prime}$ in the optimal power flow problem.

Digressing shortly, the control of the phase angle can be realized by applying devices such as a phase angle regulator or a phase angle shifter. These devices adjust the voltages and the phase angles at both sending and receiving ends of the tie-lines to establish the desired power flow. The recent advances of high capacity Flexible AC Transmission Systems (FACTS) or HVDC tie-lines enable this type of control functionality on a larger scale.

Returning to the problem at hand we are now ready to formulate the optimization model. The variables remain unchanged from subsection5.1. We need to solve:

$$
\begin{aligned}
& \min _{\mathbf{P}_{A^{\prime}}, \mathbf{P}_{B^{\prime \prime}}, \Theta_{A^{\prime}}, \Theta_{B^{\prime \prime}}} \mathbf{c}_{A^{\prime}}^{T} P_{A^{\prime}}+\mathbf{c}_{B^{\prime \prime}}^{T} P_{B^{\prime \prime}} \quad\left(\mathcal{P}_{4}\right) \\
& \text { subject to: } \\
& {\left[\begin{array}{cc}
\mathbf{A}_{A^{\prime}} & \mathbf{0} \\
\mathbf{0} & \mathbf{A}_{B^{\prime \prime}}
\end{array}\right]\left(\begin{array}{c}
\mathbf{x}_{A^{\prime}} \\
\mathbf{x}_{B^{\prime \prime}}
\end{array}\right) }=\left(\begin{array}{c}
-\mathbf{b}_{A^{\prime}} \\
-\mathbf{b}_{B^{\prime \prime}}
\end{array}\right) \\
& \Theta_{2}^{\prime}-\Theta_{4}^{\prime}=\Theta_{2}^{\prime \prime}-\Theta_{4}^{\prime \prime} \\
& \Theta_{3}^{\prime}-\Theta_{5}^{\prime}=\Theta_{3}^{\prime \prime}-\Theta_{5}^{\prime \prime} \\
& 0 \leq P_{i} \leq 300, \forall i=1, \cdots, 5 \\
& 0 \leq P_{i} \leq 300, \forall i=2, \cdots, 6 \\
&-\pi \leq \Theta_{i} \leq \pi, \quad \forall i=1, \cdots, 5 \\
&-\pi \leq \Theta_{i} \leq \pi, \quad \forall i=2, \cdots, 6 \\
& \Theta_{1}=0 \\
& \Theta_{6}=0 .
\end{aligned}
$$

For RTO A one finds the optimal solution $\left(\mathbf{P}_{A, \text { recon }}^{*}\right)^{T}=(0,0,90,0,0)$ and $\left(\boldsymbol{\Theta}_{A, \text { recon }}^{*}\right)^{T}=$ $(0,-0.036,-0.064,-0.046,-0.055)$ with an objective function value of $Z_{A, \text { recon }}^{*}=1800$. For RTO B the optimal solution is given by $\left(\mathbf{P}_{B, \text { recon }}^{*}\right)^{T}=(0,0,0,290,0)$ and $\left(\boldsymbol{\Theta}_{B, \text { recon }}^{*}\right)^{T}=(0.046,0.055,0.036,0.064,0)$ resulting in a cost of $Z_{B \text {,recon }}^{*}=11600$. The total cost to satisfy all loads is now given by $Z_{\text {recon }}^{*}=$ $1800+11600=13400$, an increase compared to the previous value of $Z_{\text {partial }}^{*}=9600$. Figure 7 displays the solution of the optimal power flow problem with reconciled flows from the perspective of RTO A. Figure 8 illustrates the solution from the perspective of RTO B. The inadvertent interchange in this example corresponds to a flow of $9 \mathrm{MW}$ over the tie-lines. We suspect that the above formulation may potentially also have applications in flow problems in other fields. Since there is no scheduled net interchange or power trade over the two regions, the result of this formulation does not have a significant impact on the economic efficiencies of both regions. Assuming that both areas have similar criteria for ancillary service procurement such as reserves, the resulting increased cost probably can be seen as the price for sharing an enhanced level of reliability realized by the interconnections and paid for by customers in both regions. Observe also that the changed flows will have implications on the pricing of previously agreed scheduled interchange between RTO A and B. In a DC model it is a well-established technique to control the power flow $T_{i, j}$ over a line $(i, j)$ by using the difference between the two phase angles $\Theta_{i}$ and $\Theta_{j}$. Here, we expand on this approach by solving two disjoint optimal power flow models in parallel while imposing similarity of flows over certain lines in both models. This formulation may potentially serve as a mathematical tool to approximate the complicated behavior of the actual physical power system. 


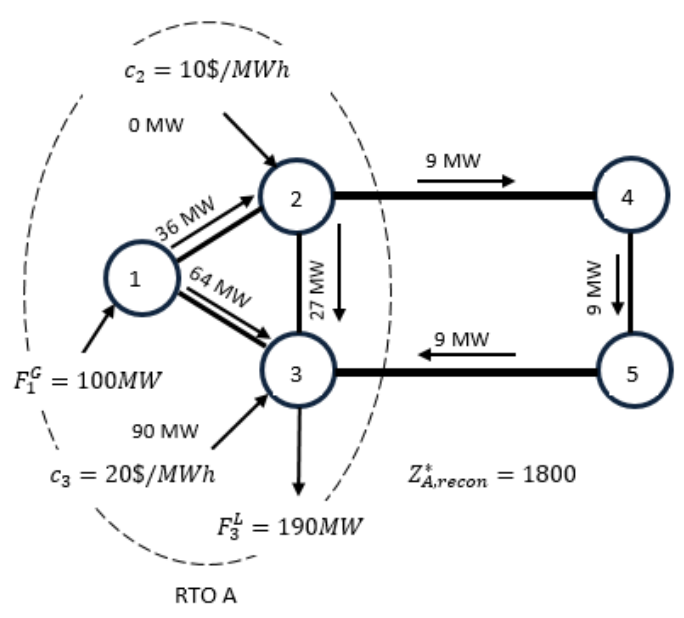

Figure 7. Reconciled flow pattern in RTO A

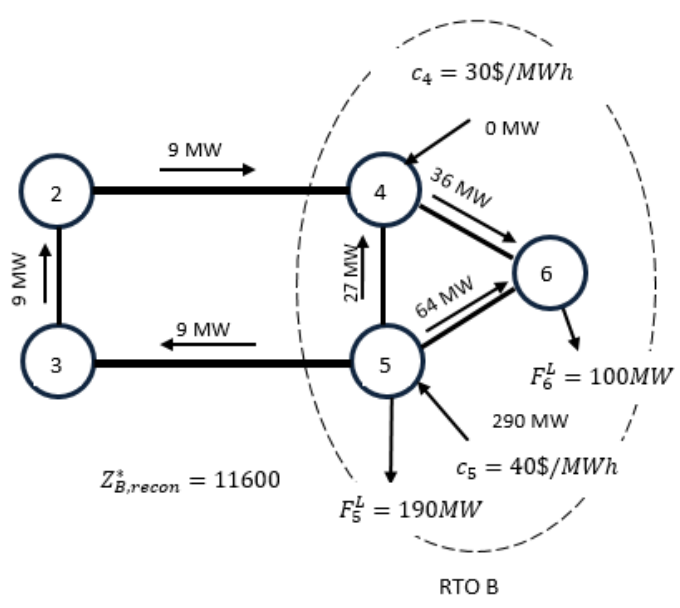

Figure 8. Reconciled flow pattern in RTO B

\section{Economic Impact on Individual RTOs}

Many research efforts have employed the joint optimal dispatch (JOD) solution as the point of reference in order to arrive at the most economic multi-area dispatch in a deregulated market environment. Specifically, two or more control areas are combined to form a new entity for which all relevant market data and transmission network parameters are available. Then the least-cost dispatch of this new "joined" total area is being computed. One challenge lies in the modeling of the information exchange between control areas. Various decomposition techniques have recently been explored to model optimal power flow over a group of fragmented power systems. In [7] and [5] Lagrangian and augmented Lagrangian techniques have been proposed. However, the required update of the multipliers is considered a disadvantage of this approach. A method referred to as optimality condition optimization (OCD) relaxes only the constraints of the neighboring control area, while the local complicating constraint is retained [8,9,10]. Here, every subproblem (control area) produces an update for the local decision variables and the local multipliers. Then these local solutions are exchanged between control areas. Extensions of this method have also been presented in $[11,12]$. A modified coordinated regional dispatch (MCRD) was investigated in $[14,13]$. The capacity of transmission lines is split between RTOs and iteratively adjusted according to the price that each RTO is willing to pay for that capacity. A marginal equivalent (ME) approach was presented in $[15,16]$. However, all of the aforementioned research efforts employed the joint optimal dispatch (JOD) as 
reference. Whether one or more control areas will be at an economic disadvantage under these conditions was not included in these investigations. With offer curves, network structure, and all capacities known for both RTO A and B, problem $\left(\mathcal{P}_{1}\right)$ will provide the JOD solution. However, in cases this solution will put one control area at a distinct disadvantage, i.e. one control area may bear the majority of the costs. How can an uneven distribution of costs to customers be avoided? In this section we propose to introduce upper bounds to limit the costs of the objective function for each control area when a joint optimal dispatch is calculated. To begin our analysis, we first separate the objective function into individual components for each RTO. For the case of two RTOs the objective function in (1) can be written as:

$$
\mathbf{c}^{T} \mathbf{P}=\mathbf{c}_{A}^{T} \mathbf{P}_{A}+\mathbf{c}_{B}^{T} \mathbf{P}_{B}
$$

Moreover, the individual objective function values for each control area can be defined as:

$$
\begin{aligned}
Z_{A} & =\mathbf{c}_{A}^{T} \mathbf{P}_{A} \\
Z_{B} & =\mathbf{c}_{B}^{T} \mathbf{P}_{B}
\end{aligned}
$$

Possible values for and may be user defined or computed using a model that minimizes cost for a single control area only. In other words, this corresponds to setting and to the objective function values of RTO A and RTO B when both RTOs are completely separated from one another. Under this ("separate") dispatch the tie-lines are disregarded and RTO A solves only for nodes 1 to 3, while RTO B solves for nodes 4 to 6 . In the following we shall use these values, labeled and and computed separately, as upper bounds for the terms and. The detailed cost vectors and the firm flows will be specified below. Using $\mathbf{P}^{T}=\left(\mathbf{P}_{A}^{T}, \mathbf{P}_{B}^{T}\right)$ and $\mathbf{c}^{T}=\left(\mathbf{c}_{A}^{T}, \mathbf{c}_{B}^{T}\right)$ we solve problem $\left(\mathcal{P}_{1}\right)$ where we include the additional constraints

$$
\begin{aligned}
& \mathbf{c}_{A}^{T} \mathbf{P}_{A} \leq Z_{A, s e p}^{*} \\
& \mathbf{c}_{B}^{T} \mathbf{P}_{B} \leq Z_{B, \text { sep }}^{*}
\end{aligned}
$$

The expressions in (30) and (31) provide a ceiling for the maximum costs incurred in RTO A or RTO B, respectively. Next, a simple example is provided for illustration. We begin by calculating the joint optimal dispatch for both RTO A and B. The JOD solution is obtained by solving problem $\left(\mathcal{P}_{1}\right)$ escribed in section 3. Note that constraints (30) and (31) are excluded for the JOD solution. The cost vector is given by $\mathbf{c}^{T}=(0,40,0,20,30,0)$. All generators are limited to a maximum output of $\bar{P}_{n}=300 M W, \forall n=1, \cdots, 6$. Firm withdrawals occur at node $1\left(F_{1}=200 M W\right)$ and node $6\left(F_{6}=300 M W\right)$, while line $(4,5)$ in RTO B has a maximum capacity of $\bar{T}_{4,5}=80 M W$. Figure 9 depicts the generation, load, and network parameters

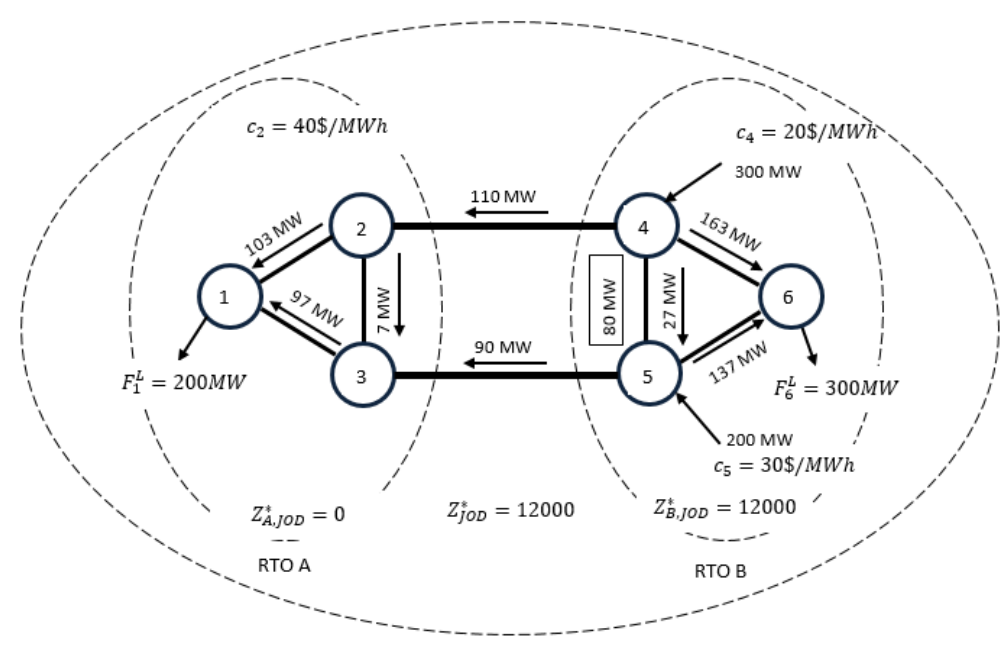

Figure 9. Flow pattern in RTO A and RTO B under JOD 
along with optimal flow resulting from the JOD solution. Specifically, the optimal dispatch is given by $\left(\mathbf{P}_{J O D}^{*}\right)^{T}=(0,0,0,300,200,0)$ and $\left(\boldsymbol{\Theta}_{J O D}^{*}\right)^{T}=(0,0.103,0.097,0.213,0.187,0.050)$, with an objective function value of $Z_{J O D}^{*}=12000$. Generation only occurs in RTO B where the cheaper generators are located operating at $20 \$ / \mathrm{MWh}$ and $30 \$ / \mathrm{MWh}$. The objective function values per area are given by $Z_{A, J O D}^{*}=0$ for RTO A and $Z_{B, J O D}^{*}=12000$ for RTO B. The 3-bus solutions, which dispatch generation for each RTO separately, result in objective function values of $Z_{A, s e p}^{*}=8000$ and $Z_{B, \text { sep }}^{*}=6300$. Observe that compared to a separate dispatch RTO B's costs increase under JOD. Moreover, consider also the costs to satisfy load. For RTO A this value is given by $12000 \frac{200}{200+300}=4800$, while for RTO B one finds $12000 \frac{300}{200+300}=7200$, well in excess of $Z_{B, \text { sep }}^{*}=6300$.

Next, we solve $\left(\mathcal{P}_{1}\right)$ including also the expressions (30) and (31), thereby limiting the costs for RTO A and RTO B. One now finds for the optimal solution $\left(\mathbf{P}_{\text {cuts }}^{*}\right)^{T}=(0,198,0,276,26,0)$ and $\left(\boldsymbol{\Theta}_{\text {cuts }}^{*}\right)^{T}=$ $(0,0.135,0.065,0.141,0.061,-0.049)$ with an objective function value of $Z_{c u t s}^{*}=14216$. Figure 10 shows



Figure 10. Flow pattern in RTO A and RTO B with bounded costs

the optimal power flows using cutting planes. Generation now occurs in both control areas and only a modest net export of $2 \mathrm{MW}$ from RTO B to RTO A can be observed over the tie-lines. Separate objective function values are given by $Z_{A, \text { cuts }}^{*}=7916$ for RTO A and $Z_{B, \text { cuts }}^{*}=6300$ for RTO B satisfying limits imposed on the costs in expression (30) and (31), since $7916 \leq 8000$ and $6300 \leq 6300$. Notice also that $Z_{\text {cuts }}^{*}=14216 \leq 14300=Z_{A, \text { sep }}^{*}+Z_{B \text {, sep }}^{*}$ Put differently, the overall costs with additional cost ceilings are lower than the sum of the individual dispatch costs. Hence, the economic burden for either of the two RTOs does not increase. Tables 3 and 4 display generation costs and customer costs in compact form. The inclusion of constraints (30) and (31) affects the optimal dispatch solution (JOD with cuts)

Table 3. Generation costs

\begin{tabular}{cccc}
\hline Model & $\begin{array}{c}\text { Generation costs } \\
\text { RTO A }\end{array}$ & $\begin{array}{c}\text { Generation costs } \\
\text { RTO B }\end{array}$ & $\begin{array}{c}\text { Generation costs } \\
\text { Total }\end{array}$ \\
\hline Separate dispatch & 8,000 & 6,300 & 14,300 \\
JOD & 0 & 12,000 & 12,000 \\
JOD with cuts & 7,916 & 6,300 & 14,216 \\
\hline
\end{tabular}

in two ways. First, the combined objective function value for both RTOs increases compared to the 
Table 4. Customer costs

\begin{tabular}{cccc}
\hline Model & $\begin{array}{c}\text { Customer costs } \\
\text { RTO A }\end{array}$ & $\begin{array}{c}\text { Customer costs } \\
\text { RTO B }\end{array}$ & $\begin{array}{c}\text { Customer costs } \\
\text { Total }\end{array}$ \\
\hline Separate dispatch & 8,000 & 6,300 & 14,300 \\
JOD & 4,800 & 7,200 & 12,000 \\
JOD with cuts & 7,956 & 6,260 & 14,216 \\
\hline
\end{tabular}

JOD-based solution. Individually however, neither RTO is worse off when compared to its respective separate dispatch. Second, these constraints also control how generation in both areas is allocated in order to satisfy load. This results in a redistribution of generation, which in turn precipitates a net scheduled interchange of $2 \mathrm{MW}$ from RTO B to RTO A.

\section{$7 \quad$ Future Research}

In section 4 several cases were presented describing how optimal power flow in one RTO is affected by the conditions in the neighboring RTO. In these instances RTO A (buses 1 to 3) had full knowledge of RTO B's grid and operating conditions. Many practitioners currently model power interchange based on proxy buses [4]. This can be interpreted as reducing all transmission grid and market information to the location of a few proxy buses with corresponding LMPs whose location may or may not coincide with the proxy buses. An analysis of the minimum amount of information required to compute a reliable dispatch solution for RTO A seems an interesting future research topic, particularly in the presence of net scheduled interchange. In section 5 a model is presented that may help reconcile power flow over edges of "quasi-disjoint" networks. The resulting solution may serve as an approximation of the inadvertent interchange between adjacent control areas. Note that the described model does not include net scheduled interchange, that is, transactions that have previously been agreed between market participants belonging to different control areas. Further analysis of the presented models might examine the effect on LMPs in both RTOs. The valuation of net scheduled interchange may be significantly affected by a change in nodal prices. The two "quasi- disjoint" optimal power flow problems are solved in parallel while imposing certain conditions on some of the phase angles. Note that the linking constraints solely focus on the differences of the angles. That is, the phase angles in each individual optimal power flow problem (prime and double prime) may assume vastly different values. Note also that these constraints impose power flow equality over identical transmission lines; hence the values of the susceptances cancel out. An examination of this approach in the context of several areas seems warranted. We also suspect that this type of "quasi-disjoint" formulation may have applications elsewhere. In section 6 a model was presented which provides insurance against a potentially uneven distribution of costs to loads under the JOD approach. Additional constraints bound the costs in each region with a value that can be controlled by each RTO. It seems natural to investigate an extension involving several regions.

\section{Concluding Remarks}

Determining the impact of dispatched generation in a power system spanning several control areas poses a variety of challenges. In this paper several models and ideas were introduced, which may assist in an improved design of interconnected multi-jurisdictional power markets. We first demonstrate that two interconnected control areas can affect each other's dispatch even when the net power interchange over the tie-lines is zero. In an example we show that the dispatching decisions in an adjacent jurisdiction can lead to congested lines and an increase in generation costs elsewhere while the net power interchange between RTOs remains zero. Furthermore, a model is presented which reconciles the tie-line flows between two control areas when both RTOs possess only limited information of each other's power system. The inclusion of the constraints imposing tie-line flow equality may help analyze the increase in total dispatch costs of both areas. In practice these increases are resolved using ad-hoc and automatic measures, which 
frequently are difficult to quantify. We also raise the concern that the current JOD-based approaches not necessarily guarantee a fair distribution of costs between adjacent control areas. Specifically, while RTO A records a decrease in generation and load costs under JOD (e.g. $Z_{A, J O D}^{*}=0 \leq Z_{A, \text { sep }}^{*}$ ), RTO $\mathrm{B}$ may observe significantly higher generation and load costs (e.g. $Z_{B, J O D}^{*}=12000 \leq Z_{B, \text { sep }}^{*}$ ). A new model is presented which may provide an insurance against uneven cost distribution by adding additional constraints that bound the costs in each control area. While the costs of the resulting model will be higher than JOD, they can be lower than the sum of the individual control area costs dispatched separately. Put differently, bounding the individual costs of each RTO will increase total costs for both RTOs compared to the JOD-based solution $\left(Z_{A, J O D}^{*}+Z_{B, J O D}^{*} \leq Z_{A, c u t s}^{*}+Z_{B, \text { cuts }}^{*}\right)$. However, feasible solutions of our proposed model will satisfy that (1) no individual RTO will be worse off compared to their individual dispatch $\left(Z_{A, \text { cuts }}^{*} \leq Z_{A, \text { sep }}^{*}\right.$ and $\left.Z_{B, \text { cuts }}^{*} \leq Z_{B, \text { sep }}^{*}\right)$ and (2) both RTOs may be better off compared to the total cost of two separate dispatches $\left(Z_{A, c u t s}^{*}+Z_{B, \text { cuts }}^{*} \leq Z_{A, \text { sep }}^{*}+Z_{B, \text { sep }}^{*}\right)$. Reconciling the tie-line flows can change the operating conditions and outcomes of two interconnected power markets, even in the absence of any economic transaction between the two regions (no scheduled net interchange). The observed effect may potentially be similar to inadvertent interchanges. Another important concern, which seems to have been overlooked in the current effort of designing interregional electricity markets, is that pursuing the JOD-based global optimal result for multiple interconnected electricity markets cannot guarantee that each market and its customers are better off.

Acknowledgments. The authors would like to acknowledge the National Science Foundation of the United States (NSF grant ECCS-955265), the National Science Foundation of China (NSFC grant E070451528701), and the Global Innovation Initiative Program of the United States and the United Kingdom for their partial support of these research activities. Theodore B. Trafalis was supported by RSF grant 14-41-00039, and he conducted research at National Research University Higher School of Economics. In addition we would like to thank G. Taylor (Brunel University, United Kingdom), C. Kang (Tsinghua University, China), K. Cheung (Alstom Grid USA, United States), T. Zheng (ISO New England, United States), B. Rew (Southwest Power Pool, United States), and Y. Li, D. Patton, and J. Chen (Potomac Economics, United States) for their helpful comments and suggestions on this research.

\section{References}

1. FERC Order, "Standard Market Design (SMD), Notice of Proposed Rulemaking (NOPR)," 2002.

2. R. Garg, "Electric transmission seams: A primer," 2015.

3. A. J. Wood and B. F. Wollenberg, Power generation, operation, and control. John Wiley \& Sons, 2012.

4. S. M. Harvey, "Proxy buses, seams and markets [draft]," NYISO Market Structures Working Group, Tech. Rep., May 2003.

5. B. H. Kim and R. Baldick, "Coarse-grained distributed optimal power flow," IEEE Transactions on Power Systems, vol. 12, no. 2, pp. 932-939, 1997.

6. R. Baldick, B. H. Kim, C. Chase, and Y. Luo, "A fast distributed implementation of optimal power flow," IEEE Transactions on Power Systems, vol. 14, no. 3, pp. 858-864, 1999.

7. A. J. Conejo and J. A. Aguado, "Multi-area coordinated decentralized dc optimal power flow," Power Systems, IEEE Transactions on, vol. 13, no. 4, pp. 1272-1278, 1998.

8. A. J. Conejo, F. J. Nogales, and F. J. Prieto, "A decomposition procedure based on approximate newton directions," Mathematical programming, vol. 93, no. 3, pp. 495-515, 2002.

9. F. J. Nogales, F. J. Prieto, and A. J. Conejo, "A decomposition methodology applied to the multi-area optimal power flow problem," Annals of operations research, vol. 120, no. 1-4, pp. 99-116, 2003.

10. A. J. Conejo, E. Castillo, R. Minguez, and R. Garcia-Bertrand, Decomposition techniques in mathematical programming: engineering and science applications. Springer Science \& Business Media, 2006.

11. A. G. Bakirtzis and P. N. Biskas, "A decentralized solution to the dc-opf of interconnected power systems," Power Systems, IEEE Transactions on, vol. 18, no. 3, pp. 1007-1013, 2003.

12. P. N. Biskas, A. G. Bakirtzis, N. I. Macheras, and N. K. Pasialis, "A decentralized implementation of dc optimal power flow on a network of computers," Power Systems, IEEE Transactions on, vol. 20, no. 1, pp. 25-33, 2005.

13. I. Midwest, "Joint operating agreement between the midwest independent transmission system operator," Inc. and PJM interconnection, LLC Available from \{www. midwestiso. org\}, 2008.

14. R. Baldick and D. Chatterjee, "Final phase i report on coordinated regional dispatch framework," 2010. 
15. F. Zhao, E. Litvinov, and T. Zheng, "A new coordination algorithm for efficient dispatch of multiarea power systems," in CIGRE Symposium, Paris, 2012.

16. — - "A marginal equivalent decomposition method and its application to multi-area optimal power flow problems," IEEE Transactions on Power Systems, vol. 1, no. 29, pp. 53-61, 2014.

17. S. M. Harvey, "TCC expansion awards for controllable devices: Initial discussion," NYISO Market Structures Working Group, Tech. Rep., February 2002.

18. ISONE and NYISO, "Inter-Regional Interchange Scheduling (IRIS) Analysis and Options," 2011.

19. P. Etingov, Y. V. Makarov, D. Wu, Z. Hou, Y. Sun, S. Maslennikov, X. Luo, T. Zheng, S. George, T. Knowland et al., "Uncertainty-based estimation of the secure range for iso new england dynamic interchange adjustment," in TESD Conference and Exposition, 2014 IEEE PES. IEEE, 2014, pp. 1-5. 